\title{
Instant lithium monitoring
}

\author{
J. R. KING, Consultant Psychiatrist, Barnsley Hall Hospital, Bromsgrove B61 0EX; \\ J.D. PHILliPS, Senior Research Fellow, School of Health Sciences, Wolverhampton \\ Polytechnic, Wolverhampton (correspondence: West Midlands Biological Psychiatry \\ Research Group, Biomedical Research Laboratory, Wolverhampton Polytechnic, \\ Wolverhampton WV1 1DJ); R. JUDGE, formerly Registrar, Barnsley Hall Hospital, \\ Bromsgrove B61 0EX; A. D. ARMOND, Consultant Psychiatrist, Burton Road \\ Hospital, Dudley, West Midlands; J. A. CoRBETT; Professor of Developmental \\ Psychiatry, Department of Psychiatry, University of Birmingham; and N. J. BIRCH, \\ Professor of Biomedical Science, Wolverhampton Polytechnic
}

Lithium therapy for affective disorders has been estimated to result in savings of hospital beds in the National Health Service of $£ 23$ million each year. In order to maintain and justify this saving we must invest to provide careful monitoring of serum lithium concentrations in patients living in the community. In recent years the advent of lithium clinics has done much to improve the compliance and safety of lithium therapy (Masterton et al, 1988), but we believe that there may be scope for further improvement both in the service to the patient and in encouragement of the patient to comply.

\section{Current problems}

Despite the most careful monitoring, there is still morbidity from the use of lithium. Toxicity arising through patient, physician or laboratory error can produce permanent renal or central nervous system damage. The reasons in the individual case are often complex, but failed communication between laboratory, doctor and patient is one potential source of error.

Typically, lithium monitoring depends on estimations performed in laboratories remote from the point of consultation, using flame emission spectroscopy or atomic absorption spectrometry. These conventional monitoring systems are cumbersome: forms have to be filled in, samples sent off to distant laboratories and the results awaited, often for several days. Urgent situations involve telephone calls to hard-pressed laboratory staff, a period of anxious waiting, and at every stage there is an opportunity for things to go wrong. Forms may be wrongly addressed, samples disappear, results may be returned to the wrong destination. Telephone calls do not get through, messages are not passed on, patients can no longer be contacted. Some method of monitoring close to the patient, which would cut through the above complexity and delay, is needed.

\section{Role of ISE analysers}

Ion selective electrode (ISE) analysers look as though they might be the answer to some of these problems, because they give an instant display and print-out of results, and most analyse whole blood, so no sample preparation is required. They work on the same principle as the $\mathrm{pH}$ meter and are now available for a range of ions. We are investigating the use of some lithium ISE analysers at the point of consultation, and have reported elsewhere preliminary experience with one such instrument at a lithium clinic (Phillips et al, 1989). Although it is early days, we anticipate that ISE analysers might be useful in three circumstances.

\section{Commencing therapy}

The most commonly used method is the simple stepwise procedure described by Schou (1986), starting at a low dose and titrating upwards to the target level. After each increment in dosage a week must be allowed for the serum level to stabilise before a blood sample can be drawn. At this point the dose should be increased but a further and unnecessary delay is imposed while the sample is sent off for analysis. Onthe-spot monitoring could halve the total time taken from the typical six weeks to only three weeks. Where acutely manic patients are concerned, the benefits would be particularly great in terms of rapid and safe stabilisation at higher serum levels.

\section{Routine maintenance therapy}

Lithium clinics are managed in a variety of ways, some of which are far from ideal. Some models are as follows.

(a) Venepuncture is performed when the patient attends. It is rare for the results to be available the same day enabling adjustments to be made at the time of consultation. More 
usually, the psychiatrist finds himself commenting on levels from the last appointment, weeks or months ago. The patient may not recall what symptoms he was experiencing at that time, what the interval between the dose and venepuncture was, or whether he remembered to take his tablet at the correct time. He is aware of his current symptoms but the psychiatrist cannot interpret these in the light of the concurrent serum level.

(b) Venepuncture is performed in advance of the clinic. With this scheme the results will be more relevant, although still a week or so out of date. However, the patient has to make a double journey to the hospital.

(c) Monitoring is undertaken jointly by general practitioner and psychiatrist. The patient attends the surgery for venepuncture and the psychiatrist for more detailed supervision. This scheme depends on close co-operation between GP and psychiatrist and, in practice, there is plenty of room for confusion (Masterton et al, 1988). For example, the results may be returned to the general practitioner instead of the psychiatrist or, where both receive the result, the patient may be given conflicting advice. Again, such advice will be based on the situation a week or more previously. On-the-spot monitoring offers a way of eliminating these problems. The result is more meaningful and patients learn it at first hand, enhancing their sense of involvement in the treatment. Full involvement in treatment, enabling the patient to accept some measure of responsibility for it, is now recognised to be of paramount importance for compliance and safety (Schou, 1986). Also, faced with immediate feedback, potentially non-compliant patients are less likely to 'forget' to take their medication. In this way, the basic aims of the lithium clinic are furthered.

\section{On-demand monitoring}

Random monitoring by conventional methods is of limited effectiveness in identifying intercurrent problems. Elevated lithium levels due to fluctuations of sodium balance, illness or interaction from other drugs are unlikely to coincide obligingly with the times of routine monitoring, even when this is carried out as frequently as three monthly. Acknowledging this, Johnson (1987) has advocated flexibility in the monitoring frequency, which may be once a year or even less in stable patients. This does not, however, prevent problems arising and perhaps increases the need for an on-demand service, which can rapidly identify emergent problems as they arise and obviate the need for lithium to be discontinued while conventional analysis takes place. The period of anxious waiting while the patient's problems are sorted out is drastically reduced, saving medical time, increasing patient satisfaction and benefiting compliance.

\section{Potential drawbacks}

It is clear that these new instruments offer many advantages. However, as with most new technologies, there are problems. The analysers are expensive, costing from $£ 5000-£ 7000$. Although running costs are small at 25 estimations per day (36p per sample), they rise steeply to $1.67 \mathrm{p}$ per sample at five estimations a day. They therefore need to be fully used in order to be cost effective.

Although they are easy to operate, experience with other instruments sited outside laboratories suggests that they are reliable only if supervised by laboratory staff. The best place to site them is open to discussion and the practicability of regularly carrying them to and from lithium clinics has yet to be confirmed.

Finally, ISE analysers need more field-testing. Nevertheless, we believe that they have a potential value which is worth exploring further.

\section{References}

Jornson, F. N. (1987) In Depression and Mania: Modern Lithium Therapy (ed. F. N. Johnson), p. 111. Oxford: IRL Press.

MASTERTON, G., WARNER, M. \& ROXBURGH, B. (1988) Supervising lithium. A comparison of a lithium clinic, psychiatric out-patient clinics and general practice. British Journal of Psychiatry, 152, 535-538.

Phillips, J. D., KING, J. R., Myers, D. H. \& BirCh, N. J. (1989) Lithium monitoring close to the patients. Lancet, ii, 1461 .

SCHOU, M. (1986) Lithium treatment: a refresher course. British Journal of Psychiatry, 149, 541-547. 